G U E S T E D I T O R I A L

\title{
Special Section on Color Imaging: Processing, Hardcopy, and Applications
}

\author{
Reiner Eschbach \\ Xerox Corporation \\ Digital Imaging Technology Center \\ 800 Phillips Road, 128-27E \\ Webster, New York 14580 \\ E-mail: eschbach@crt.xerox.com
}

\author{
Gabriel Marcu \\ Apple Computer, Inc. \\ Graphics and Imaging \\ 1 Infinite Loop, MS 305-1DE \\ Cupertino, California 95014 \\ E-mail: marcu@apple.com
}

This special section offers a sample of the recent research results in the area of color and image processing, spreading from theoretical research to practical algorithms and their hardware implementation, from image acquisition to signal processing and transmission.

The first paper, "Analysis of tristimulus interdifference and contextual color correction," by Rizzi, Gadia, and Marini, discusses the possibility of extrapolation of the spatial compensation performed by a human observer when judging color into the area of digital image processing. The authors prove that the interdifference between tristimulus colors obtained using different proposed color matching functions indeed decreases when the spatial information and contextual color correction are used. One of the applications of this research is in the synthetic image generation of scenes under different illuminants for rendering on displays.

The optimization of colors for display rendering is presented in the second paper, "Preferred skin color reproduction on the display," by Park et al. The paper describes a methodology to find the regions of optimal memory colors and proposes a local adjustment technique for optimizing the image color quality, following the idea that the correct reproduction of the memory color is the most important criterion for maintaining the naturalness of the image. The parameters of the proposed technique are derived from psychophysical experiments using real images and tailored to color reproduction on displays. The technique is applied for optimizing the skin color reproduction on displays, one of the most critical elements in judging the color quality of a TV set.

The third paper, "Colorimetric characterization of scanner by measures of perceptual color error," by Shen, Mou, and Xin, proposes two scanner characterization methods based on color error minimization between the scanned color and a color target. The method proves to be more accurate than the conventional CIEXYZ-space-based characterization methods.

The last paper, "Transmission of images over general packet radio services using advanced wavelet image codecs and digital signal processor chips," by Gleich, Planinšič, and Mohorko, is an application of image processing into a codec design for optimization of coding and signal transmission. The codec architecture allows image capture from the camera, wavelet-based image coding, and image transmission through a wireless network. The paper presents practical design constraints, such as the tradeoff between complexity and efficiency of a wavelet-based image coder, and issues concerning wavelet transform and entropy coding. The compatibility with JPEG2000 shows that on this par- ticular implementation, the proposed coding method is significantly faster.

These papers prove once again the advances of color and image processing in recent years. They add to the dynamic advances in electronic imaging, and publishing these results in this journal contributes to the recording and distribution of technical information in the technical community. They also constitute an invitation to submit more papers in the field of color and image processing to the Journal of Electronic Imaging.

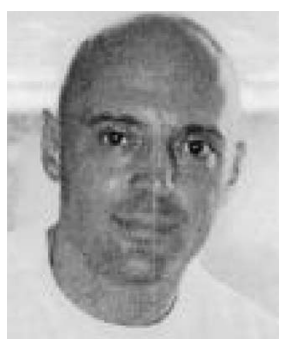

Reiner Eschbach received his MS and $\mathrm{PhD}$ in physics from the University of Essen, Germany, in 1983 and 1986, respectively. From 1986 to 1988 he was a visiting scholar at the University of California, San Diego. He joined Xerox in 1988 where he became a principal scientist at the Xerox Digital Imaging Technology Center in 1994 and a research fellow in 2000. Dr. Eschbach is a member of SPIE and a fellow of the Society for Imaging Science \& Technology (IS\&T). He is the former editor of the IS\&T Recent Progress series as well as past secretary and publications vice president of IS\&T. He is also a former member of the SPIE Publications Committee. $\mathrm{He}$ is an associate editor for the IEEE Transactions on Image Processing in the areas of scan- 
ning, display, and printing. His research interests include color image processing, digital halftoning, and compression.

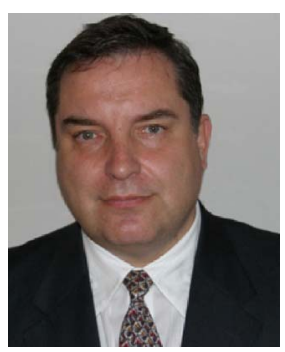

Gabriel Marcu is a senior scientist at Apple Computer Inc. He received a DiplEng in 1982 and a Dr. degree in 1991 , both in electronics engineering, from the Polytechnic Institute of Bucharest, Romania. He was then with the Research Institute of Computers in Bucharest and with Graphica Computer Corporation and Array Corporation in Japan working on various projects on image and color processing. In
1996 he joined Apple Computer. Since then, his responsibilities have included display and printer color reproduction. His achievements are in color reproduction on displays and printers, including characterization/ calibration, halftoning, gamut mapping, ICC profiling, and color quality. Dr. Marcu has taught seminars and short courses on color topics for Shizuoka University, Japan, the University of California at Berkeley, and at various IS\&T, SPIE, and Society for Information Display conferences. 